\title{
NIR observations of dEs in the Virgo cluster: a structural continuity with giant ellipticals
}

\author{
Stefano Zibetti ${ }^{1}$ and the GOLDMiNe Research Team ${ }^{2}$ \\ ${ }^{1}$ Max-Planck-Institut für Extraterrestrische Physik \\ Gießenbachstraße, D-85748, Garching bei München, Germany - email: szibetti@mpe.mpg.de \\ ${ }^{2}$ http://goldmine.mib.infn.it/
}

\begin{abstract}
The structural properties of a sample of 50 dEs in the Virgo cluster are here derived from Near InfraRed (NIR, H-band $1.65 \mu \mathrm{m}$ ) surface photometry and analysed. One-dimensional surface brightness (SB) profiles are extracted using elliptical isophote fitting. They are characterised by means of structural parameters, namely the half light radius $R_{e}$, the average surface brightness within $R_{e}\left(\mu_{e}\right)$, and a concentration index $\left(c_{31}\right)$. We show that typical dEs have close-to-exponential NIR SB distributions.

The relations between dEs and giant ellipticals (Es) are investigated by comparing the NIR structural parameters of 273 Es in nearby clusters. Further analysis is conducted using the optical-NIR colour $B-H$ and by studying the relationships between structural and dynamical parameters (fundamental plane) for the two classes of galaxies. The transition between the two regimes is smooth and no dichotomy is seen.
\end{abstract}

Keywords. galaxies: dwarf; elliptical and lenticular, cD; fundamental parameters; structure; kinematics and dynamics; galaxies: clusters: individual (Virgo, Coma, A1367)

\section{Introduction and sample}

Dwarf elliptical galaxies are not just a rescaled version of their giant counterparts. Optical observations have shown that, as opposed to Es, dEs have exponential rather than de Vaucouleurs SB profiles, and do not follow the same scaling relations linking luminosity, the effective radius $R_{e}$ and the mean effective surface brightness $\mu_{e}$.

In this work we re-assess the structural properties of dEs using NIR surface photometry, which is a good tracer of the bulk of the stellar mass, while being much less affected than optical bands by dust attenuation and metallicity effects. This project is part of a large NIR survey of galaxies in nearby clusters (Gavazzi et al. 2000, 2003). This has allowed to compare the derived properties with those of $\mathrm{E}$ galaxies in the full range of luminosities.

The $50 \mathrm{dEs}$ of this work have been selected from the VCC (Binggeli, Sandage \& Tammann, 1985), limited to photographic magnitude $m_{\mathrm{p}}<16$, and observed at the $3.6 \mathrm{~m}$ telescopes ESO/NTT and TNG (Gavazzi et al. 2001). Our sample is representative of $\sim 30 \%$ of the dEs more luminous than $10^{8.5} \mathrm{~L}_{\odot}(\mathrm{H}) .48$ out of these 50 have B-band photometry available, and 6 have also velocity dispersion measurements. 273 Es (including 35 in Virgo and the complete sample of 217 Es in Coma and Abell 1367) have been used to investigate the relations between dwarves and giants.

\section{Results}

The SB profile of each galaxy is extracted by fitting elliptical isophotes with standard IRAF procedures. The decomposition of the 1-D profile with simple analytical functions (exponential, de Vaucouleurs and their combinations) is used to extrapolate an asymptotic magnitude (extrapolations are of the order of 0.1-0.2 mag). From the measured 

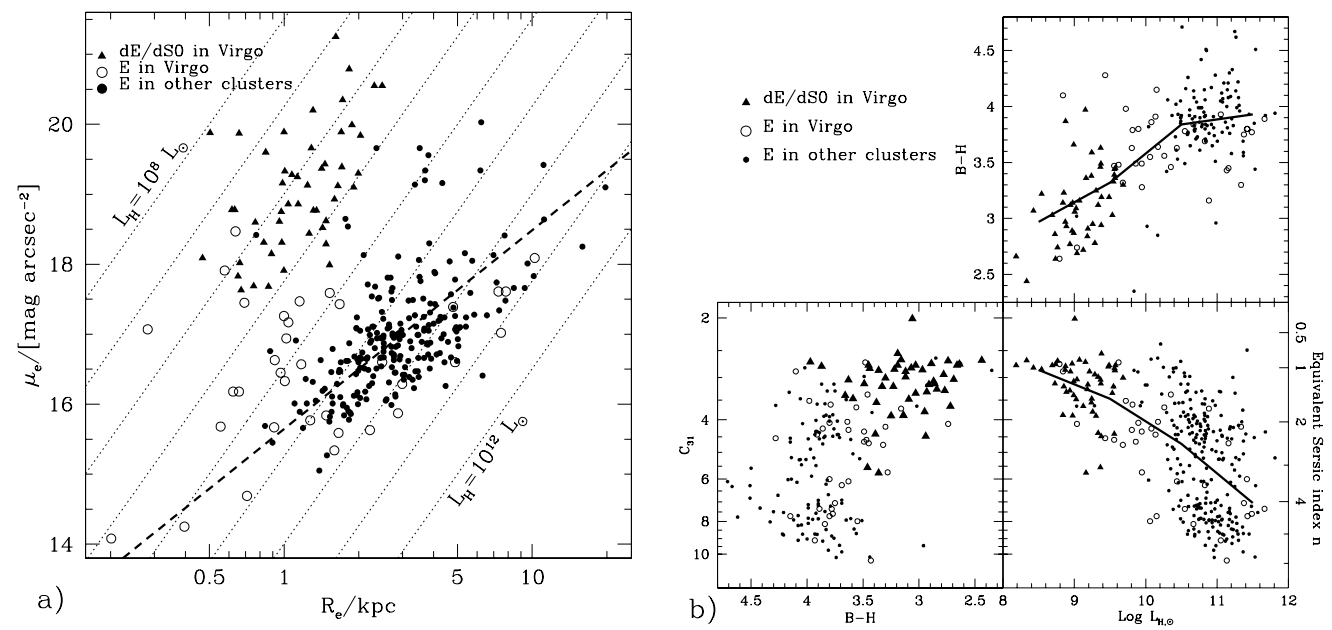

Figure 1. a) The $H$-band $\mu_{e}-R_{e}$ plane. The thick dashed line is the "Kormendy" relation for the Es. Dotted lines are isoluminosity lines assuming a fixed ellipticity $=0.35$. b) The "photometric cube", alias $c_{31}-(B-H)-L_{H}$ space. Trends are indicated by the thick lines, representing the median of $c_{31}$ and $B-H$ in 4 bins of $L_{H}$. The right-side scale of the $c_{31}-L_{H}$ panel reports the value of the Sérsic index $n$ that reproduces the same $c_{31}$.

$S B$ profile we extract the effective radius $R_{e}$ enclosing half of the asymptotic flux, and the average SB $\mu_{e}$ within $R_{e}$. The distribution of dEs and Es in this plane (Fig. 1a) does not display any significant gap between the two populations. The "Kormendy" relation for Es (thick dashed line) represents the upper limit for the SB at given $R_{e}$, with dEs having lower SB than Es. The multi-dimensional photometric parameter space $c_{31}-(B-H)-L_{H} \dagger$ (Fig 1b, see also Scodeggio et al. 2002) shows very clearly the smooth transition from blue, low-concentration (nearly exponential) dEs to red, concentrated (roughly de Vaucouleurs) Es.

We have analysed the Fundamental Plane relations for the galaxies with a velocity dispersion measure. Using NIR structural parameters, dEs lie on the same relations as Es. Systematic variations of $M / L$ in the NIR have been investigated by means of the $\kappa$ space formalism (Bender, Burstein \& Faber, 1992): although a trend with mass is clearly seen at high masses, dEs span the same range in $M / L$ as low-mass Es. Adopting a more sophisticated formalism, in which profile deprojection is used to take non-homology and spectroscopic aperture effects into account (Zibetti et al. 2002), we show that the data are consistent with constant $M / L$ throughout the sequence of Es and dEs.

\section{References}

Bender, R., Burstein, D. \& Faber, S.M. 1992, ApJ 399, 462

Binggeli B., Sandage A. \& Tammann G.A. 1985, AJ 90, 1681

Gavazzi, G., Franzetti, P., Scodeggio, M., Boselli, A. \& Pierini, D. 2000, A\&A 361, 863

Gavazzi, G., Zibetti, S., Boselli, A., Franzetti, P., Scodeggio, M. \& Martocchi, S. 2001, A\&A 372,29

Gavazzi, G., Boselli, A., Donati, A., Franzetti, P. \& Scodeggio, M. 2003, A\&A 400, 451

Scodeggio, M., Gavazzi,G., Franzetti,P., Boselli,A., Zibetti,S. \& Pierini,D. 2002, A $\& A$ 384, 812

Zibetti, S., Gavazzi, G., Scodeggio, M., Franzetti, P. \& Boselli, A. 2002, ApJ 579, 261

$\dagger$ The concentration index $c_{31}$ is the ratio of the radii enclosing $75 \%$ and $25 \%$ of the asymptotic flux. 